\title{
Zur Datierung der Begleitkeramik der Glockenbecherkultur
}

\section{To dating the common ware of the Bell Beaker Culture}

\author{
Zoja Benkovsky-Pivovarová
}

\begin{abstract}
Abstrakt
Im Beitrag wird auf die Begleitkeramik der Glockenbecherkultur aus Niederösterreich aufmerksam gemacht, die in der Literatur unter Aunjetitz-Kultur veröffentlicht wurde. Aufgrund der Radiokarbondaten aus Mähren wird gezeigt, dass die Begleitkeramik in den erwähnten Gebieten sich beim gegenwärtigen Forschungsstand nicht genau datieren lässt und dass die bisherige Gliederung der Glockenbecherkultur in Mähren nicht aufrechterhalten werden kann. Diese Daten unterstützen die Meinung von J. S. Shennan aus dem Jahre 1975, nach welcher das Inventar der Gräber der Glockenbecherkultur in Mitteleuropa eher auf den sozialen Status der Verstorbenen als auf die chronologischen Unterschiede zurückzuführen ist. Für die Erörterung der Fragen der Feinchronologie sind weitere Serien an Radiokarbondaten notwendig.
\end{abstract}

\section{Schlagwörter}

Glockenbecherkultur, Begleitkeramik, Niederösterreich, Radiokarbondaten, Mähren

\begin{abstract}
The paper draws attention to the Bell Beaker common ware from Lower Austria, which was published in literature under the Únětice Culture. The radiocarbon dates from Moravia revealed that the common ware from the above-mentioned territory cannot be dated with accuracy and that the existing division of the Bell Beaker Culture in Moravia cannot be maintained. These dates support the 1975 opinion by J. S. Shennan, according to which the grave finds of the Bell Beaker Culture in Central Europe refer to social status of the deceased rather than to chronological differences. The ongoing discussion on the problems of detailed chronology requires another series of radiocarbon dates.
\end{abstract}

\section{Keywords}

Bell Beaker Culture, common ware, Lower Austria, radiocarbon dates, Moravia 
In diesem Beitrag wird auf einige Funde der Glockenbecherkultur aus Niederösterreich eingegangen, die in der Literatur der Aunjetitz-Kultur zugeschrieben wurden. Zu ihrer Datierung wird auf der Grundlage von Radiokarbondaten aus Mähren Stellung genommen.

Das Grab von Hetzmannsdorf wurde im Jahre 2000 von V. Heyd in die Phase B2a der späten Glockenbecherkultur (Spätkupferzeit 3) gestellt (Heyd 2000, 12, Taf. 107, 119). In diesem Grab war eine adulte, etwa 30-50 Jahre alte, S-N orientierte Frau in rechter Hockerlage und mit typischem Inventar der Glockenbecherkultur - Knochenknöpfen mit V-Bohrung, einer ritzverzierten Tasse und einem Topf - bestattet (Abb. 1). Die Lage und Orientierung der Toten ist nicht nur für die Frauengräber der Glockenbecherkultur (Havel 1978, 95; Dvořák 1993, 226, 532; Neugebauer 1994, 38), sondern - mit Abweichungen - auch für die Toten beider Geschlechter in der mährisch-niederösterreichischen Aunjetitz-Kultur charakteristisch (Stuchlik 1993, 245-246; Neugebauer 1994, 113; Lauermann 1995, 80); es war vermutlich diese Tatsache, die zur ursprünglichen Zuordnung des Grabes zur Aunjetitz-Kultur führte (Hasenhündl 1991, 193194, Abb. 387-389; Lauermann 2003, 131, Abb. 59). Die Knochenknöpfe mit V-Bohrung, die in der Glockenbecherkultur als Kleidungsbesatz, Knöpfe und als Halsketten bzw. Teile von diesen gedient haben könnten (Schwarz 2008, 115; Matějićková - Dvořák 2012b, 253; 2012c, 269270, Abb. 10, 11), sind in Mähren hauptsächlich in Frauengräbern der Glockenbecherkultur anzutreffen (Dvořák 1993, 226; Matějićková 2009, 162; Matějičková - Dvořák 2012b, 253); so sind sie z. B. im größten Gräberfeld der Glockenbecherkultur in Mähren - in Hoštice I - in 24 von insgesamt 157 Gräbern vorhanden, wobei es sich in 18 Fällen um Frauengräber handelt, die 3 - 33 Knöpfe enthielten (Matějićková - Dvořák 2012b, 253; 2012c, 269-270, Abb. 10, 11 - wohl eher mit flacher Seite nach unten eingefädelt!).
Diese Art von Knochenknöpfen ist auch noch in frühbronzezeitlichen Kulturen von Frankreich bis in den Donauraum vorhanden (Krause 1988, 98; Czebreszuk - Makarowicz 1993, Abb. 1), was in der Mitte des 20. Jh. auf das Weiterleben der Glockenbecherkultur (Guyan 1949-1950, 182-184; Hájek 1950, 357; Hetzer 1949, 95; Felgenhauer 1952, 20), die Wurzeln der frühbronzezeitlichen Kulturen in der inzwischen untergegangenen Glockenbecherkultur (Vladár 1964, 113) und die Existenz der frühbronzezeitlichen Kulturen im Karpatenbecken zur Zeit des Äneolithikums in Böhmen (Hájek 1957, 420; Korrektur der Ansichten aus 1950) zurückgeführt wurde. Es ist beachtenswert, dass in Niederösterreich solche in die Frühbronzezeit zu datierenden Knochenknöpfe hauptsächlich in der Unterwölbling-Kultur Verwendung fanden (Reitberger 2008, 83-84), d. h. in einer Kultur, die ihre Toten in der für die Glockenbecherkultur typischen „bipolaren“ Lage bestattete, während aus dem Verbreitungsgebiet der Aunjetitz-Kultur nach wie vor nur die Bernsteinperle mit V-Bohrung aus dem Grab 8 von Laa/ Thaya bekannt ist, dessen Keramikinventar sich aus Elementen der Glockenbecher- und der Aunjetitz-Kultur zusammensetzt (Beninger 1933, 174; Felgenhauer 1952, 15; Kern 2003b, Abb. 1-3; Benkovsky-Pivovarová 2009, 247-248); die Perlen dieser Art stellen den charakteristischen Typ der Bernsteinperlen in der Glockenbecherkultur dar (Czebreszuk - Makarowicz 1993, 530).

Die Tasse von Hetzmannsdorf (Abb. 1) findet verlässliche Entsprechungen in den Grabfunden von Blažovice II und Opatovice (Dvořák 1992, Taf. 40-C:1; 62-C:4) wie auch im Siedlungsgut von Brno-Obřany (Ondráček - Dvořák Matějićková 2005, Taf. 32:217, 218 u. w.). Für die Grube 2/79 von Holubice, die zwei vergleichbare, aber unverzierte Tassen enthielt, sind Radiokarbondaten mit divergierenden Angaben vorhanden (Bln-2841: Rakovský 1985, 386; Görsdorf 1993, 112; Peška 2009, 249; Ondráček 

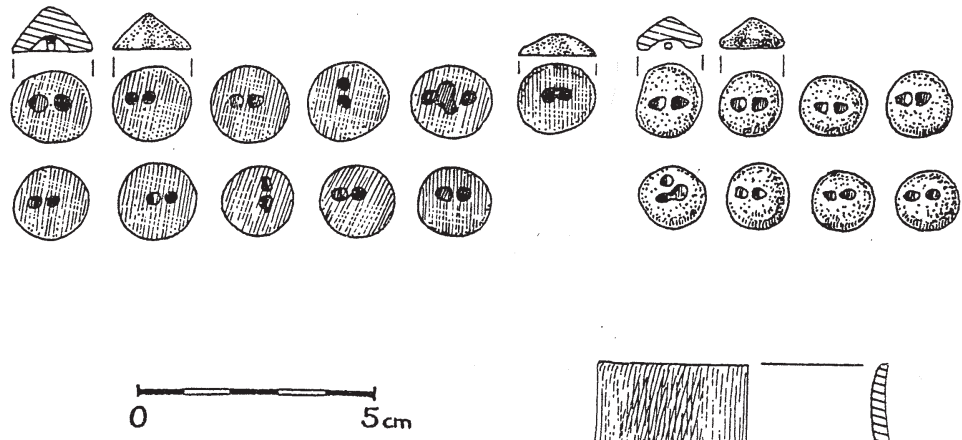

+ Scherben eines Topfes

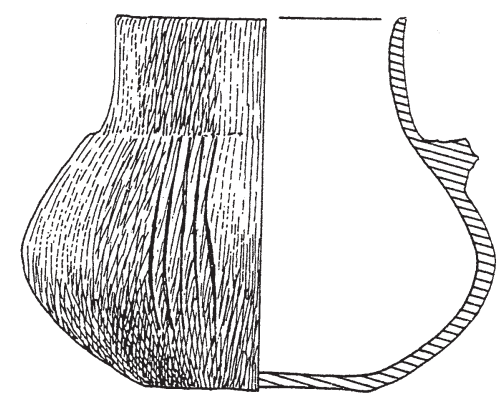

Abb 1. Hetzmannsdorf. (Nach Hasenhündl 1991)

Fig. 1. Hetzmannsdorf (after Hasenhündl 1991)

- Dvořák - Matějićcová 2005, Taf. 72:39, 40). Nach K. Rassmann, der sich auf das etwas ältere bei J. Görsdorf angeführte Datum stützte, ist dieses teilweise mit der älteren Aunjetitz-Kultur von Prasklice parallelisierbar (Rassmann 1996, 203, Abb. 4; dazu Benkovsky-Pivovarová 2009, 245-246), was dem durch Radiokarbondaten nachgewiesenen Überdauern der Glockenbecherkultur in Mitteleuropa in die frühe Bronzezeit entspricht (Rassmann 1996, Abb. 2, 6; Müller 1999, Abb. 12); dieses wurde jüngst auch durch die Daten für das Gräberfeld von Pavlov I nachgewiesen (Peška 2009, Abb. 53, 54). Auch beim Grab von Hetzmannsdorf ist im Lichte der bereits zitierten Radiokarbondaten für die Grube 2/79 von Holubice eine Datierung in die früheste Bronzezeit denkbar.

Aus einem Körpergrab von Poysdorf stammt eine Tasse mit in der Standfläche eingeritztem Kreuz (Abb. 2:2; Scheibenreiter 1953, 13, Taf.
62:4). An Parallelstücken seien die Tassen von Klobouky und Zwingendorf/Alicenhof genannt (Dvořák - Matějićková - Peška - Rakovský 1996, Taf. 34-A:2; Kern 2000-2001, Taf. 7-16:2). Poysdorf ist seit Langem als Fundort eines Töpfchens der Glockenbecherkultur bekannt (Franz - Hesch - Menghin - Mitscha-Märheim 1924, 25; Hetzer 1949, Abb. 20:13). Unter dem Fundortsnamen Langenlois bildete F. Scheibenreiter vier der insgesamt fünf im Naturhistorischen Museum in Wien aufbewahrten Gefäße aus einem im Jahre 1888 beim Bau der Kamptalbahn angeschnittenen Gräberfeld ab (Abb. 2, 5; Scheibenreiter 1953, 113, Taf. 48:9-12; Lauermann 2003, 117, Abb. 83). Die übrigen Funde aus diesem Gräberfeld sind leider verschollen, aber auch das fünfte im Naturhistorischen $\mathrm{Mu}$ seum aufbewahrte Gefäß - der Teil einer Tasse wie etwa Abb. 2:4, aber mit abgesetztem Hals - gehört der Glockenbecherkultur an. Bei der 

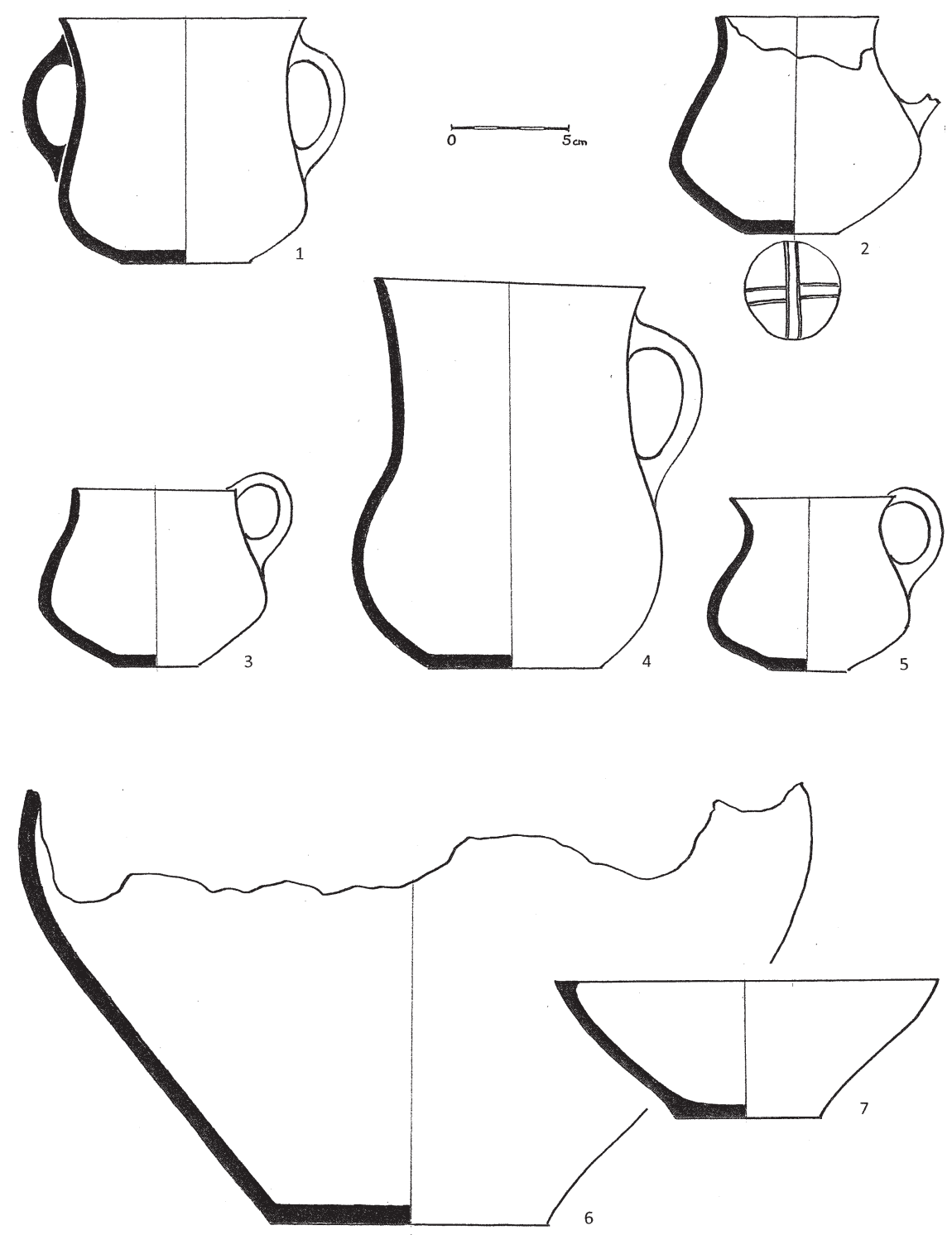

Abb 2. 1, 4 - Stillfried; 2 - Poysdorf; 3, 5-7 - Haindorf. (Nach Scheibenreiter 1953)

Fig. 2. 1, 4 - Stillfried; 2 - Poysdorf; 3, 5-7 - Haindorf. (after Scheibenreiter 1953) 
Fundortsangabe richtete sich der Autor nach $I$. Spöttl (1890, 85), aber im Jahre 1949 wurden diese Funde von K. Hetzer unter dem Fundortsnamen Haindorf kartiert (Hetzer 1949, Abb. 20:16), unter welchem sie nach einer dankenswerten Auskunft von W. Antl-Weiser im Naturhistorischen Museum in Wien auch inventarisiert sind. Die Schüssel (Abb. 2:7) repräsentiert einen charakteristischen Schüsseltyp der Glockenbecherkultur (Typ H1: Ondráček - Dvořák - Matějićková 2005, 13) und auch die Tassen (Abb. 2:3,5) sind für diese Kultur kennzeichnend (Dvořák - Hájek 1990, Taf. XX:3, XXV-A:1, XXVIII-B:4; Dvoŕák 1992, Taf. 12-C:3, 36:29, 60A:1; Dvořák - Matějićcková - Peška - Rakovský 1996, Taf. 4:19,20,31, 21-A:3, 58-B:1; Stuchlik Stuchliková 1996, Abb. 31:7; Matějićková 2012, Taf. 24-825:4; 121-817:2). Ebenfalls ein Krug und eine kleine Amphore von Stillfried (Abb. 2:1,4; Scheibenreiter 1953, 70, Taf. 82:19,20) finden gute Vergleichsmöglichkeiten im Keramikgut der Glockenbecherkultur (Dvořák - Hájek 1990, Taf. XVIII:3; Dvořák 1992, Taf. 59-A:10; Dvořák - Peška 1993, Abb. 8-B:2; Dvořák Matějićcková - Peška - Rakovský 1996, Taf. 2-E:1; Cheben - Drahošová 2004, Abb. 2:10; Matejićková 2012, Taf. 47-864:2 u. w.).

Bei allen erwähnten Gefäßen von Hetzmannsdorf, Haindorf, Poysdorf und Stillfried handelt es sich um die Begleit- bzw. Komplementärkeramik der Glockenbecherkultur (Strahm 2004, 103; zu den Termini Begleitkeramik und Komplementärkeramik Kern 2003a, 253). In einer Übersicht der Ansichten zur Gliederung der Glockenbecherkultur zeigte J. Turek, dass sowohl die in Böhmen verwendete dreistufige Gliederung, als auch die in Mähren verwendete ursprünglich ebenfalls dreistufige, nunmehr vierstufige Gliederung dieser Kultur in der zweistufigen Gliederung der österreichischen Fundbestandes der Glockenbecherkultur durch R. Pittioni aus dem Jahre 1954 verankert ist (Turek 2008, 150-151). Nach R. Pittioni gehören der älteren Stufe in erster Linie die Glockenbecher und die Armschutzplatten, der jüngeren Stufe die triangulären Dolche, die Dolche mit kurzer Griffangel, die konischen Schüsseln mit vier Füßchen, die kugeligen Henkeltassen, die Beinknöpfe mit V-Bohrung und die Anhänger aus Eberhauern an (Pittioni 1954, 252, 261, 265). Gemäß der Gliederung der Glockenbecherkultur Mährens durch P. Dvořák, die in ihrer älteren dreistufigen Konzeption auch in Niederösterreich angewendet wird (z. B. Neugebauer 1994, 37; Neugebauer - Neugebauer 1997, 309; Kern 2000-2001, 317; Penz 2010, 23f; Preinfalk - Preinfalk - Artner - Müller 2015, 83), kommen aber nur die Fundgruppen I/II II und III P. Dvořáks infrage; die Fundgruppe I wird durch große und schlanke Glockenbecher repräsentiert, die Fundgruppe I/II enthält außer der Glockenbecher auch die Begleitkeramik heimischen und innerkarpatischen Ursprungs, in der Fundgruppe II nimmt die Anzahl der Becher ab, während die der Begleitkeramik ansteigt und für die Fundgruppe III ist das fast gänzliche Fehlen der Becher und die plastische sowie geritzte Verzierung der Keramik charakteristisch (Dvořák 1993, 224, 532; Matějícková - Dvořák 2012a, 133). An charakteristischen Formen der Begleitkeramik werden in der Fundgruppe I/II jene mit Csepel-Nagyréver-Merkmalen, ferner Krüge vom Somogyvár-Typ, Amphoren und amphorenartige Gefäße sowie Schüsseln mit hohem Hals genannt, bei der Fundgruppe II soll es sich um eine lokale Entwicklung auf unbekannter Grundlage handeln und die Fundgruppe III wird für eine Fortsetzung der Fundgruppe II gehalten (Ondráček - Dvořák Matějićková 2005, 16). Im Jahre 2005 wurde von J. Ondráček, P. Dvořák und A. Matějićcková (2005, 16) auf die Unsicherheit der chronologischen Abfolge der genannten Fundgruppen in Mähren hingewiesen, aber nach der Veröffentlichung der Radiokarbondaten von Hoštice I und Záhlinice I wird diese von A. Matějíčková 
und P. Dvořák doch für wahrscheinlich gehalten (Matějičková, Dvořák 2012a, 133).

Beim gegenwärtigen Forschungsstand stehen zwar nur relativ wenige Radiokarbondaten für die Glockenbecherkultur Mährens zur Verfügung, dennoch tragen diese zur Datierung der Begleitkeramik bei. Die in Wien und Erlangen erarbeiteten Radiokarbondaten für Pavlov I divergieren bedauerlicherweise in mehreren Fällen (Peška 2009, Abb. 54, 55), weswegen in der Abb. 3 nur die Erlangener Daten berücksichtigt werden; die Funde aus diesem Gräberfeld wurden von P. Dvořák, A. Matějičková, J. Peška und $I$. Rakovský (1996, Taf. 37-B, 42-F, 47-B) veröffentlicht. Die Funde von Záhlinice I publizierten $P$. Dvořák, I. Rakovský und J. Stuchliková (1994), die von Hoštice I A. Matějićková (2012); die in der Abb. 3 angeführten VERA-Daten von den beiden erwähnten Fundorten wurden von J. Peška (2012, 153-165, Abb. 1, 4, 5) zusammengestellt und ausgewertet. Die Daten für fünf Bestattete im Grab H2/91 von Tvořihráz wurden wegen der großen Zeitspanne (Görsdorf 1999, 71-78; Peška 2009, 249) und das Datum für das Grab 937 von Hoštice I wegen des Vorkommens eines mit Begleitkeramik vergesellschafteten unverzierten Bechers (Matějićková 2012, 174, Taf. 87:5) außer Acht gelassen.

Die Zusammenstellung von nicht kalibrierten Daten für 15 Gräber mit Keramik von den drei erwähnten Gräberfeldern (Abb. 3) zeigt, dass die ältesten Gräber - mit relativ großem Abstand von den übrigen Gräbern - die nur Begleitkeramik enthaltenden Gräber 516 und 501 von Pavlov I sind; sie sind älter, als das Grab 48 mit einem Krug innerkarpatischen Herkunft von Záhlinice I, bei dem eine Datierung in die Fundgruppe I/II erwogen wurde (Dvořák - $R a$ kovský - Stuchliková 1994, 221). Die nur Glockenbecher enthaltenden Gräber (Pavlov I, Gr. 570, Záhlinice I, Gr. 47) sind annähernd gleichzeitig nicht nur mit den Gräbern, die Glockenbecher und Begleitkeramik enthielten (Pavlov I,
Gr. 585; Záhlinice I, Gr. 48; Hoštice I, Gr. 837, 947, 826), sondern auch mit nur Begleitkeramik enthaltenden Gräbern (Pavlov I, Gr. 500, 505; Záhlinice I, Gr. 5; Hoštice I, Gr. 937). Zwei Gräber mit Begleitkeramik (Záhlinice I, Gr. 67; Hoštice I, Gr. 820) schließen diese Datenserie ab. Für das birituelle Grab 570 von Pavlov I mit Glockenbechern sind zwei Erlangener Daten mit unterschiedlichen Die sich aus den nicht kalibrierten Daten ergebenden Schlüsse werden durch die kalibrierten Daten bestätig (Peška 2009, Abb. 56; 2021, Abb. 2, 3)

Obwohl an der Zahl relativ gering, bestätigen die bis jetzt zur Verfügung stehenden nicht kalibrierten Radiokarbondaten von Hoštice I, Pavlov I und Záhlinice I die bereits erwähnte Skepsis von J. Ondráček, P. Dvořák und A. Matějičková $(2005,16)$ hinsichtlich der Zeitabfolge der Fundgruppen I, I/II, II und III der Glockenbecherkultur Mährens. Die Datenserien aus zwei Laboratorien (Erlangen und Wien) brachten ähnliche Resultate in Bezug auf die etwa gleichzeitige Existenz der Gräber mit Glockenbechern, der Gräber mit Glockenbechern sowie Begleitkeramik und der Gräber mit Begleitkeramik. Im gegebenen Rahmen unterstützen sie die von $\mathrm{S}$. J. Shennan bereits im Jahre 1975 geäußerte Meinung über die Gleichzeitigkeit der Glockenbecher und der Begleitkeramik in Mitteleuropa; die Gründe für die Ausstattung der Gräber mit Inventar wären seiner Meinung nach im sozialen Status der Verstorbenen zu suchen (Shennan 1975). Mit sozialer Struktur der Ostgruppe der Glockenbecherkultur setzte sich neuerlich M. Schwarz auseinander; sie behielt dabei die traditionelle Gliederung dieser Gruppe in drei Stufen bei (Schwarz 2008, 16-17, Tab. 1.1).

Die Angaben zu den oben erwähnten Fremdelementen in der Fundgruppe I/II der Glockenbecherkultur Mährens wären um die Keramikfunde der Kosihy-Čaka/Makó-Kultur 
$\square \mathrm{GB} \quad \square \mathrm{GB}+\mathrm{BK} \quad \square \mathrm{BK}$

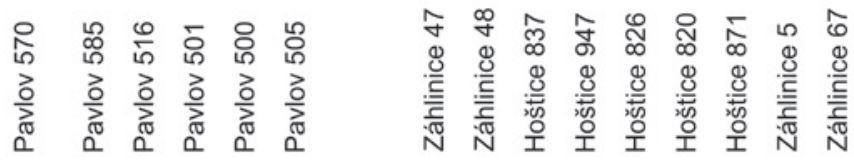

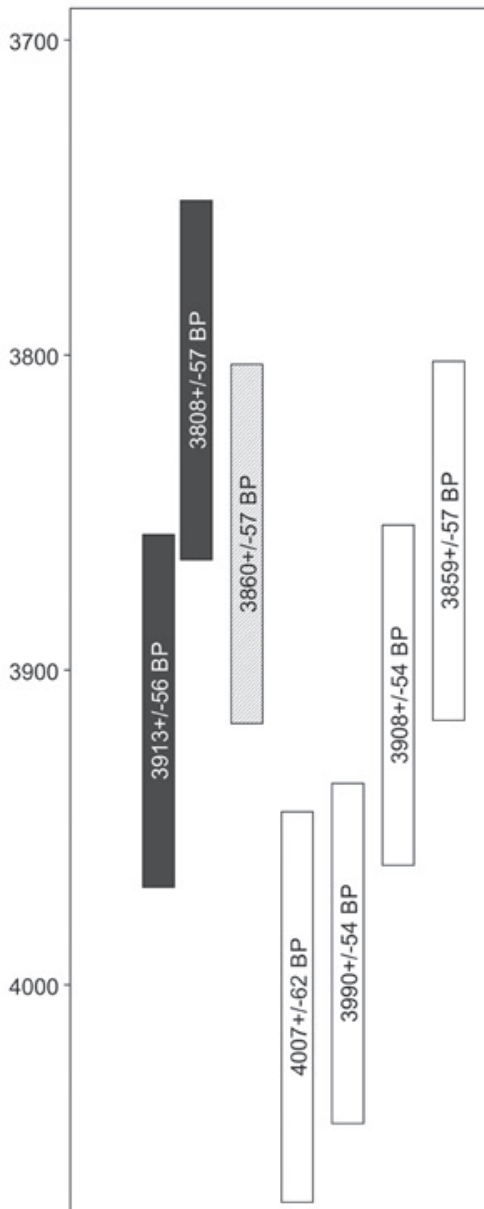

a

잉
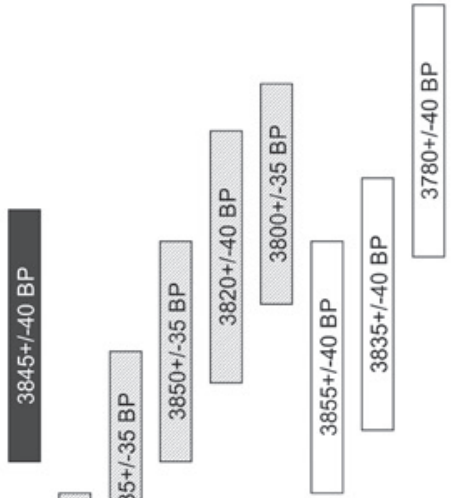

离

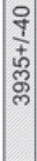

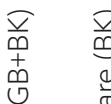

还要

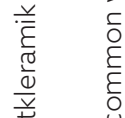

$\frac{0}{\sqrt[0]{4}}$

๓

$\frac{0}{3} \quad \frac{1}{2}$

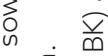

ㄷ. 둘

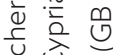

过.

(1) $\ddot{v}$

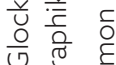

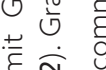

-

()

둥일

过

o $\overline{\overline{0}}$

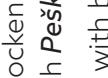

ए

产 $\frac{c}{\bar{Q}}$

㐫苋

तi

(.)

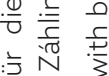

进 N

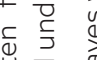

पू

诺

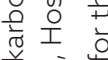

응

实

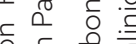

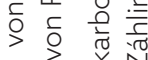

O)

亏 妾 要

离站造过

है

ह

जू के

సू

$\dot{m} \stackrel{\vec{E}}{\dot{m}}$

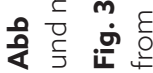


zu ergänzen, auf die V. Moucha aufmerksam machte (Moucha 1981, 115-117). Die Ansichten über die Datierung der Kosihy-Čaka/Makó-Kultur und die Frage ihres Verhältnisses zur Glockenbecherkultur divergieren. Während es sich nach einigen ungarischen Autoren um zwei nacheinander folgende Kulturen handelt, wobei die Glockenbecherkultur für jünger gehalten wird (z. B. Schreiber-Kalicz 1984, Tab. auf S. 169; Bóna 1992, Tab. auf S. 40; Kalicz-Schreiber 1995, Abb. 15; Kalicz-Schreiber - Kalicz 1998-1999, Abb. 20), sind die diesbezüglichen Meinungen weiterer Autoren im Sinne von zwei gleichzeitig existierenden oder nacheinander folgenden Kulturen uneinheitlich (z. B. Lichardus - Vladár 1998, Abb. 5; Bátora 2000, Abb. 692; Bertemes 2000, Abb. 5; Peška 2001, Abb. 1; Bertemes Heyd 2002, Abb. 8; Endrödi 2003, 267; Kopacz Šebela 2006, Abb. 1; Peška 2009, 242; Matějićcková - Dvoŕák 2012a, Abb. 1-2; Horváth - Dani Petô - Pospieszny - Svingor 2013, Tab. 6). Von G. Kulcsár wird die Kosihy-Čaka/Makó-Kultur zwischen 2900/2700 und 2500/2300 datiert (Kulcsár 2009, 15). Nach V. Moucha sind die Keramik typen der Kosihy-Čaka/Makó-Kultur nicht nur in den Gräbern mit Glockenbechern wie z. B. im Grab XII von Šlapanice I, sondern auch in den Gräbern von Moravský Krumlov I, Slavkov u Brna I, Šlapanice I und Ledce I, die nur Begleitkeramik enthielten, vorhanden. Besonders hervorzuheben ist das Grab XI von Šlapanice I (Moucha 1981, Taf. 1:1-4, 2:8-11, Taf. 3), in welchem ein charakteristisches Gefäß der Kosihy-Čaka/Makó-Kultur vom Typ VII/20 nach G. Kulcsár (Moucha 1981, Taf. 1:1; Dvořák - Hájek 1990, Taf. XVII-B:1; Kulcsár 2009, Abb. 23) mit Tassen vom Typ D1 und D2 sowie einer Schüssel vom Typ H1 der Glockenbecherkultur nach J. Ondráček - P. Dvořák - A. Matějičková
(2005, 12-13) bzw. mit Tassen vom Typ 34 und einer Schüssel vom Typ 78 nach M. Besse (2003 nach Limburský 2012, Abb. 67) vergesellschaftet war. In diesem Grab erscheint also ein typisches Gefäß der Kosihy-Čaka/Makó-Kultur zusammen mit vollentwickelter, charakteristischer Begleitkeramik der Glockenbecherkultur.

Das Auftreten von verzierten Glockenbechern bis in die Zeit der Protoaunjetitz-Kultur ist durch die Zusammensetzung des Grabinventars des Körpergrabes von Strážnice gesichert. In diesem Grab waren zwei Erwachsene und ein Kind bestattet; die Erwachsenen waren mit Kopfausrichtung nach SW, das zwischen ihnen liegende Kind mit Kopfausrichtung nach NO bestattet; bei den Füßen eines der Erwachsenen lag ein verzierter Glockenbecher, zwischen den Skeletten waren Tierknochen deponiert, die mit einer typischen, mit Blindhenkeln und einem Bandhenkel versehenen Schüssel der Protoaunjetitz-Kultur bedeckt waren (Skácel 1955). Es ist m. W. der einzige Fall eines solchen Grabinventars in Mähren; bei den von J. Ondráček (1967, 404) als Analogien für die Schüssel aus diesem Grab angeführten Exemplaren der Glockenbecherkultur handelt es sich um Stücke mit kehligem Rand und Bandhenkeln ohne Blindhenkel.

Die Ausführungen zur Datierung der Begleitkeramik der Glockenbecherkultur können mit der bereits im Jahre 1989 von V. Moucha geäußerten Meinung abgeschlossen werden, dass die Schwierigkeiten bei der Periodisierung der Glockenbecherkultur in Böhmen auf die Stabilität dieser Kultur zurückzuführen sind (Moucha 1989, 213). Für die Erörterung feinchronologischer Fragen sind daher weitere Serien von Radiokarbondaten notwendig. 


\section{Literaturverzeichnis}

Bátora, J. 2000: Das Gräberfeld von Jelšovce/Slowakei. Ein Beitrag zur Frühbronzezeit im nordwestlichen Karpatenbecken, Prähistorische Archäologie in Südosteuropa 16.

Beninger, E. 1933: Laa a d. Thaya, BH Mistelbach. In: Caspart, J., Fundberichte 1932, Fundberichte aus Österreich I, 174.

Benkovsky-Pivovarová, Z. 2009: Zur Verwandschaft einiger Keramikformen der Glockenbecherkultur und des Mad'arovce/Věteřov/BöheimkirchenKulturkreises, Pravěk, NŘ 18, 241-260.

Bertemes, F. 2000: Zur Entstehung der Danubischen Frühbronzezeit in Mitteleuropa. In: Pavlů, I. (Hrsg.), In memoriam Jan Rulf, Památky archeologické, Supplementum 13, 25-37.

Bertemes, F. - Heyd, V. 2002: Der Übergang Kupferzeit/ Frühbronzezeit am Nordwestrand des Karpatenbeckens - kulturgeschichtliche und paläometallurgische Betrachtungen. In: E. Pernicka - M. Bartelheim Krause, R. (Hrsg.), Die Anfänge der Metallurgie in der Alten Welt, Forschungen zur Archäometrie und Altertumswissenschaft 1, 187-222.

Besse, M. 2003: L`Europe du $3^{\text {e }}$ millénaire avant notre ère: les céramique commune au Campaniforme. Lausanne.

Bóna, I. 1992: Bronzezeitliche Tell-Kulturen in Ungarn. In: W. Meier-Arendt (Hrsg.), Bronzezeit in Ungarn. Forschungen in Tell-Siedlungen an Donau und Theiss. Frankfurt am Main, 9-41.

Czebreszuk, J. - Makarowicz, P. 1993: The problem of amber buttons with $\mathrm{V}$-shaped perforation in the Bell Beaker Culture. In: Actes du XIIe Congrès International des Sciences Préhistoriques et Protohistoriques, Bratislava, 1-7 Septembre 1991, 2, Bratislava, 529-532.

Dvoŕák, P. 1992: Die Gräberfelder der Glockenbecherkultur in Mähren I (Bez. Blansko, Brno-město, Brno-venkov). Brno.

Dvořák, P. - Hájek, L. 1990: Die Gräberfelder der Glockenbecherkultur bei Šlapanice (Bez. Brnovenkov). Katalog der Funde, Mährische Archäologische Quellen. Brno.

Dvořák, P. - Matějićková, A. - Peška, J. - Rakovský, I. 1996: Gräberfelder der Glockenbecherkultur in Mähren II (Bezirk Břeclav). Brno-Olomouc.
Dvořák, P. - Peška, J. 1993: Příspěvek k poznání kultury se zvoncovitými poháry na Moravě, Časopis Moravského Muzea 78, 1/2, 29-49.

Duořák, P. - Rakouský, I. - Stuchliková, J. 1994: Pohřebiště lidu se zvoncovými poháry u Záhlinic, okr. Kroměříž, Pravěk NŘ 2, 215-232.

Endródi, A. 2003: The Late Phase of the Bell BeakerCsepel Group in Hungary. British Archaeological Series, International Series 1155, 265-276.

Felgenhauer, F. 1952: Frühbronzezeitliche Gräber aus Spitz a. d. Donau, N.-Ö. Ein Beitrag zum Problem des Typus Unterwölbling, Archaeologia Austriaca XI, 1-25.

Franz, L. - Hesch, M. - Menghin, O. - Mitscha-Märheim 1924: Die prähistorische Sammlung des Niederösterreichischen Landesmuseums. Materialien zur Urgeschichte Österreichs 2, Wien.

Görsdorf, J. 1993: 14C-Datierungen des Berliner Labors zur Problematik der chronologischen Einordnung der frühen Bronzezeit in Mitteleuropa. In: Rassmann, K., Spätneolithikum und frühe Bronzezeit im Flachland zwischen Elbe und Oder, Beiträge zur Ur- und Frühgeschichte Mecklenburg-Vorpommerns 28, 97-117.

Görsdorf, J. 1999: Výsledky radiokarbonového datování. Ergebnisse der Radiokarbondatierung. In: Bálek, M. - Dvořák, P. - Kovárník, J. - Matějíčková, A., Pohřebiště kultury zvoncovitých pohárů v Tvořihrázi (okr. Znojmo). Pravěk - Supplementum 4. Brno, 71-78.

Guyan, W. U. 1949-1950: Beitrag zur Datierung einer jungsteinzeitlichen Gräbergruppe im Kanton Schaffhausen, Jahrbuch der Schweizerischen Gesellschaft für Ur- und Frühgeschichte, 163-192.

Hájek, L. 1950: Bronzové (mědené?) jehlice kultury zvoncovitých pohárů, Obzor praehistorický XIV1, 353-359.

Hájek, L. 1957: Knoflíky středoevropské skupiny kultury zvoncovitých pohárů, Památky archeologické XLVIII, 389-424.

Hasenhündl, G. 1991: KG Hetzmannsdorf, MG Wullersdorf, VB Hollabrunn, Fundberichte aus Österreich 29, 193-194.

Havel, J. 1978: Pohřební ritus kultury zvoncovitých pohárů v Čechách a na Moravě, Praehistorica VII, Varia Archaeologica 1, 91-117. 
Hetzer, K. 1949: Beiträge zur Kenntnis der Glockenbecherkultur in Österreich, Archaeologia Austriaca $4,87-131$.

Heyd, V. 2000: Die Spätkupferzeit in Süddeutschland, Saarbrücker Beiträge zur Archäologie 73, 2000.

Horváth, T. - Dani, J. - Petö, A. - Pospieszny, L. - Svingor, É. 2013: Multidisciplinary Contributions to the Study of Pit Grave Culture Kurgans of the Great Hungarian Plain. In: Heyd, V. -Kulcsár, G. - Szeverényi, V. (eds.), Transitions to the Bronze Age. Interregional Interaction and Socio-Cultural Change in the Third Millennium BC Carpathian Basin and Neighbouring Regions, 153-180.

Cheben, I. - Drahošová, V. 2004: Nálezy kultúry zvoncovitých pohárov zo Skalice. In: Lutovský, M. (ed.), Otázky neolitu a eneolitu 2003, Sborník referátů z 22. pracovního setkání badatelů zameřených na výzkum neolitu a eneolitu. Český Brod-Kounice 23. až 26. září 2003. Praha, 355-357.

Kalicz-Schreiber, R. 1995: Bronzkori urnatemetö Szigetszentmiklós határában, Ráckevei Múzeumi Füzetek 2, 5-169.

Kalicz-Schreiber, R. - Kalicz, N. 1998-1999: A Somogyvár-Vinkovci kutúra és a Harangedény-Csepelcsoport Budapest kora bronzkorában (Die Somogyvár-Vinkovci-Kultur und die Glockenbecher in der Frühbronzezeit von Budapest), Savaria 24/3, Pars archeologica, 83-115.

Kern, D. 2000-2001: Endneolithisches Gräberfeld mit Glockenbechern von Zwingendorf/Alicenhof, VB Mistelbach, Niederösterreich, Archaeologia Austriaca LXXXIV-LXXXV, 307-328.

Kern, D. 2003a: Glockenbecherkultur in Ostösterreich - andere Fragen andere Antworten? In: Czebreszuk, J. - Schmyt, M. (ed.), The Northeast Frontier of Bell Beakers. Proceedings of the symposium held at the Adam Mickiewicz University, Poznań (Poland), May 26-29 2002, British Archaeological Report, International Series 1155, 249-254.

Kern, D. 2003b: Überlegungen zum Nachweis von Mobilität und Migration in Ostösterreich zur Zeit der Glockenbecher, Mitteilungen der Anthropologischen Gesellschaft in Wien CXXXIII, 73-83.

J. Kopacz, L. Šebela, 2006: Kultura unietycka i grupa wieterzowska na Morawach na podstawie materiałów krzemieniarskich, Kraków-Brno.
Krause, R. 1988: Die endneolithischen und frühbronzezeitlichen Grabfunde auf der Nordstadterasse von Singen am Hohentwiel. Forschungen und Berichte zur Vor- und Frühgeschichte in BadenWürttemberg 32.

Kulcsár, G. 2009: The beginnings of the Bronze Age in the Carpathian Basin. The Makó-Kosihy-Caka and the Somogyvár-Vinkovci cultures in Hungary, Varia Archaeologica Hungarica XXIII.

Lauermann, E. 1995: Ein frühbronzezeitliches Gräberfeld aus Unterhautzenthal, NÖ. Stockerau.

Lauermann, E. 2003: Studien zur Aunjetitz-Kultur im nördlichen Niederösterreich, Universitätsforschungen zur prähistorischen Archäologie 99.

Lichardus, J. - Vladár, J. 1998: Frühe und mittlere Bronzezeit in der Südwestslowakei. Forschungsbeitrag von Anton Točík (Rückblick und Ausblick), Slovenská archeológia XLV-2, 221-352.

Limburský, P. 2012: Pohřebiště kultury se zvoncovitými poháry ve Vliněvsi. K problematice a chronologii konce eneolitu a počátku doby bronzové, Dissertationes Archaeologicae Brunensis/Pragensesque 13, 2012.

Matějićková, A. 2009: Pohřebiště kultury zvoncovitých pohárů z Brankovic, okr. Vyškov, Pravěk, NŘ 18, 153-164.

Matějićcková, A. 2012: Pohřebiště kultury zvoncovitých pohárů na trase dálnice DI Vyškov - Mořice. Pravěk - Supplementum 24, svazek II. Brno.

Matějićková, A. - Dvořák, P. (eds.) 2012: Pohřebiště z období zvoncovitých pohárů na trase dálnice D1 Vyškov - Mořice. Pravěk - Supplementum 24, svazek I. Brno.

Matějićková, A. - Dvořák, P. 2012a: Relativní chronologie období KZP na Moravě a pohřebiště Hoštice I. In: Matějíčková - Dvořák 2012, 133-146.

Matějićková, A. - Dvořák, P. 2012b: Kostěné artefakty z Hoštic I a Ivanovic VI v kontextu KZP na Morave. In: Matějíčková - Dvořák 2012, 253-267.

Matějičková, A. - Dvořák P. 2012c: Rekonstrukce možného využití knoflíků s V-vrtáním. In: Matějíčková - Dvořák 2012, 269-270.

Moucha,V. 1981: Südöstliche Elemente in der mährischen und böhmischen Gruppe der Glockenbecherkultur. In: Kalicz N. - Kalicz-Schreiber, R. (Hrsg.), Die Frühbronzezeit im Karpatenbecken und in den Nachbargebieten. Internationales Symposium 
1977 Budapest-Velem, Mitteilungen des Archäologischen Instituts der Ungarischen Akademie der Wissenschaften, Beiheft 2. Budapest, 115-123.

Moucha, V. 1989: Böhmen am Ausklang des Äneolithikums und am Anfang der Bronzezeit. In: Das Äneolithikum und die früheste Bronzezeit (C ${ }^{14} 3000-2000$ b. c.) in Mitteleuropa: Kulturelle und chronologische Beziehungen. Praehistorica XV, 213-218.

Müller, J. 1999: Zur absolutchronologischen Datierung und Interpretation der mitteldeutschen $\mathrm{Au}$ njetitz-Inventare. In: Bátora, J. - Peška, J. (Hrsg.), Aktuelle Probleme der Erforschung der Frühbronzezeit in Böhmen und Mähren und in der Slowakei, Archaeologia Slovaca Monographiae I, 113-126.

Neugebauer, J.-W. 1994: Frühe Bronzezeit. In: Neugebauer, J.-W., Bronzezeit in Ostösterreich, Wissenschaftliche Schriftenreihe Niederösterreich 98/99/199/101. St. Pölten-Wien.

Neugebauer, Ch. - Neugebauer, J.-W. 1997: Zum Forschungstand der Glockenbecherkultur in Niederösterreich. In: Tradition und Innovation. Prähistorische Archäologie als historische Wissenschaft, Festschrift für Christian Strahm, Internationale Archäologie, Studia honoraria 3, 307-324.

Ondráček, J. 1967: Moravská protoúnětická kultura, Slovenská archeológia XV-2, 1967, 389-446.

Ondráček, J. - Duoráke, P. - Matějićčková, A. 2005: Siedlungen der Glockenbecherkultur in Mähren. Katalog der Funde. Pravěk - Supplementum 15.

Penz, M. 2010: Eine Siedlungsgrube der späten Glockenbecherkultur aus Wien 3, Rennweg 16 (Vorbericht). Fundort Wine 13/10, 210, 20-31.

Peška, J. 2001: Das Besiedlungsbild des Spätneolithikums und der Frühbronzezeit in Südmähren. In: Lippert, A. - Schultz, M. - Shennan, S. - TeschlerNicola, M. (Red.), Mensch und Umwelt während des Neolithikums und der Frühbronzezeit in Mitteleuropa, Internationaler Workshop vom 9.-12. November 1995 Wien. Internationale Archäologie 2. Arbeitsgemeinschaft, Symposium, Tagung, Kongress, Rahden/Westf., 139-148.

Peška, J. 2009: Protoúnětické pohřebiště z Pavlova, Olomouc.

Peška, J. 2012: Absolutní datování hrobů z období KZP z Hoštic I a ze Záhlinic I (Absolute Dating of the BB graves from Hoštice I and Záhlinice). In: Dvořák - Matějiččková 2012, 153-165.

Pittioni, R. 1954: Urgeschichte des österreichischen Raumes. Wien.

Podborský, V. a kol. 1993: Pravěké dějiny Moravy. Vlastivěda moravská. Země a lid. Nová řada, svazek 3. Brno.

Preinfalk, A. - Preinfalk, F. - Artner, G. - Müller, S. 2015: Die Siedlungskammer am Nordrand der Falkensteiner Berge. In: Preinfalk et al., Trassenarchäologie II. Neue Grabungen im nördlichen Weinviertel, Fundberichte aus Österreich, Materialhefte A, Sonderheft 23, 72-91.

Rakovský, I. 1985: Sídliště kultury zvoncovitých pohárů v Holubicích (A Bell Beaker culture site at Holubice), Archeologické rozhledy XXXVII, 377-389.

Rassmann, K. 1996: Zum Forschungsstand der absoluten Chronologie der Frühbronzezeit in Mitteleuropa auf der Grundlage von Radiokarbondaten, Acta Archaeologica Kopenhagen 67, 199-209.

Reitberger, M. M. 2008: Das frühbronzezeitliche Gräberfeld von Haid, Oberösterreich, Studien zur Kulturgeschichte von Oberösterreich 20.

Shennan, S. J. 1975: Die soziale Bedeutung der Glockenbecher in Mitteleuropa. Acta Archaeologica Carpathica 15, 1975, 173-179.

Scheibenreiter, F. 1953: Die Aunjetitz-Kultur in Niederösterreich, unveröffentlichte Dissertation. Wien.

Schreiber-Kalicz, R. 1984: Komplex der Nagyrév-Kultur. In: Tasić, N. (Hrsg.), Kulturen der Frühbronzezeit des Karpatenbeckens und Nordbalkans, Beograd, 133-190.

Schwarz, M. 2008: Studien zur Sozialstruktur der Ostgruppe der Glockenbecherkultur, Saarbrücker Beiträge zur Archäologie 85.

Skácel, J. 1955: Stopy kultury zvoncovitých pohárů ve Strážnici, Studie Krajského muzea v Gottwaldově 4, 1955, 14-16.

Spöttl, J. 1890: Resultate der Ausgrabungen für die Anthropologische Gesellschaft in Niederösterreich und in Mähren im Jahre 1898, Mitteilungen der Anthropologischen Gesellschaft XX, N. F. 10, 59-100.

Strahm, Ch. 2004: Das Glockenbecher-Phänomenon aus der Sicht der Komplementär-Keramik. In: J. Czebreszuk (ed.), Similar but Different. Bell Beakers in Europe. Poznań, 101-126. 
Stuchlik, S. 1993: Únětická kultura. In: Podborský, V. Turek, J. 2008: Kultura zvoncovitých pohárů. In: Neus kolektivem 1993, 238-257.

Stuchlik, S. - Stuchliková, J. 1996: Pravěká pohřebiště stupný, E. (ed.), Archeologie pravěkých Čech 4. Eneolit. Praha, 147-169.

v Moravské Nové Vsi - Hruškách. Studie Ar- Vladár, J. 1964: Vplyvy kultúry zvoncovitých pohárov cheologického ústavu Akademie věd ČR v Brně $16-1$. v náplni nitrianskej skupiny, Študijné Zvesti Archeologického ústavu SAV 13, 111-126. 


\section{K datování doprovodné keramiky kultury se zvoncovitými poháry}

Na úvod př́spěvku je poukazováno na nálezy kultury se zvoncovitými poháry z Haindorfu, Hetzmannsdorfu, Poysdorfu a Stillfriedu (obr. 1, 2), které byly publikovány jako nálezy únětické kultury (Scheibenreiter 1963, 17, 70; Hasenhündl 1991, 193-194; Lauermann 2000, 131); nálezy z Haindorfu K. Hetzer v roce 1964 uvedl mezi nálezy kultury zvoncovitých pohárů (Hetzer 1964, Abb. 20:16) a hrob z Hetzmanddsdorfu V. Heyd v roce 2000 zařadil do fáze B2a kultury se zvoncovitými poháry (Heyd 2000, 12, Taf. 107, 119). S výjimkou hrobu z Hetzmannsdorfu se jedná o nálezy sběrového charakteru. Ve světle absolutně datovaných nálezů z jámy $2 / 79$ z Holubic, která obsahovala dva nezdobené šálky podobného tvaru, ale nelze vyloučit datování uvedeného hrobu do období starší únětické kultury.

Ve všech uvedených nálezech se jedná o doprovodnou resp. komplementární keramiku (k terminologii: Strahm 2004, 103; Kern 2003a, 253) kultury se zvoncovitými poháry. Datování a původ této keramiky je za současného stavu bádání nevyřešený. Dokazují to radiokarbonová data ze tří lokalit na Moravě (obr. 3). Dvě série dat, vypracované v laboratořích v Erlangenu a ve Vídni, dokazují, že hroby, které v keramické inventáři obsahovaly pouze zvoncové poháry (GB), hroby, které obsahovaly jak zvoncovité poháry, tak i doprovodnou keramiku $(\mathrm{GB}+\mathrm{BK})$ a hroby, které obsahovaly pouze doprovodnou keramiku (BK), jsou víceméně současné; toto zjištění se samozřejmě týká jen těch typů zvoncovitých pohárů a doprovodné keramiky, které jsou v těchto hrobech zastoupené. Nejstaršími hroby v těchto dvou datových sérií, jsou dva hroby, které obsahovaly pouze doprovodnou keramiku (Pavlov I, hroby 501 a 516), tzn., že uvedené hroby z Pavlova i. Jsou starší než hrob 47 ze Záhlinic I, který byl kvưli džbánu karpatského původu zařazený do stupně I/II (Dvořák - Rakovský - Stuchliková 1994, 221). Radiokarbanová data tedy potvrzují pochybnosti moravských autorů o následnosti stupňů I, I/II, II a III této kultury na Moravě (Ondráček Dvořák - Matějićcková 2005, 16) a podporují názor S. J. Shennana z roku 1975 o současnosti zvoncovitých pohárů a doprovodné keramiky ve střední Evropě; podle tohoto autora je inventář hrobů této kultury ve střední Evropě determinovaný sociálním postavením mrtvých (Shennan 1975). Těmito otázkami se nejnověji zabývala $\mathrm{M}$. Schwarzová, která východní skupinu kultury zvoncovitých pohárů rozdělila do tři stupňů (Schwarz 2008, 16-17, tab. 1.1). Dlouhou životnost zdobených zvoncovitých pohárů přesvědčivě dokazuje i inventář trojhrobu ze Strážnice, ve kterém se kromě poháru vyskytla i typická mísa protoúnětické kultury (Skácel 1955).

V. Moucha již v roce 1989 poukázal na problémy př̀i periodizaci kultury zvoncovitých pohárů v Čechách, které vyplývají z její statickosti (Moucha 1989, 213). Ǩešení otázek jemné chronologie této kultury je za této situace vázané na další série radiokarbonových dat. 


\section{Dr. Zoja Benkovsky}

- Donaustr. 89/5, 2344 Maria Enzersdorf-Südstadt

zoja.benkovsky@gmail.com 